\title{
Pediatric Blunt Trauma and REBOA
}

\author{
Alexis D Smith MD \\ Children's Healthcare of Atlanta, Emory University, USA
}

This is a landmark case report in that it is the first described pediatric blunt trauma patient presenting in cardiac arrest with no signs of life that survived to discharge under the age of 14 . Aortic occlusion in adolescents and children has long been debated due to dismal outcomes, especially in pediatric blunt trauma in which only two survivors who presented in cardiac arrest have ever been described and no survivors documented under the age of 14 [1,2]. Current recommendations for aortic occlusion in children are extrapolated from adult data and are still highly debated. Some trauma surgeons support its utilization in only traumatically injured children that present with signs of life while others advocate for its removal altogether from the trauma treatment algorithm [1].

This paper now demonstrates that a severely injured child due to blunt injury could benefit from endovascular occlusion of the aorta as an adjunct in resuscitation for temporization of hemorrhage. The use of REBOA in the pediatric population was first described in 2010 in an acute care surgical patient by vascular surgeons at Emory with deployment to control a ruptured aortoesophageal fistula in a 10-year-old child [3]. They utilized a 7F sheath and $14 \mathrm{~mm}$ angioplasty balloon and were able to successfully temporize hemorrhage and bridge the patient to endovascular stent placement. A survey of Japanese experience with REBOA in pediatric trauma patients was recently published, which reviewed 54 cases of the use of REBOA in trauma patients under the age of 18 [4]. However, the Japanese database failed to capture any REBOA-specific details such as hemodynamic status, timing of AO, size of catheters or complications.

During a recent Pediatric Trauma Society meeting, the US series on pediatric and adolescent REBOA (youngest age of 14, mean age of 17) described REBOA-specific details in 7 patients that underwent endovascular aortic occlusion. While their overall in-hospital mortality was $71 \%$; all of those patients were in arrest at the time of REBOA with ongoing CPR, had return of spontaneous circulation (ROSC), and survived to the operating room. The primary critique of the study was on the lack of change in paradigm as there was no survivor to discharge of pediatric blunt trauma due to utilization of the REBOA. Therefore, this case report of the first survivor of blunt pediatric trauma under the age of 14 with the use of aortic occlusion may have the potential to have a large impact on the consideration of REBOA in the pediatric and adolescent trauma population.

\section{REFERENCES}

[1] Duron V, Burke RV, Bliss D, et al. Survival of pediatric blunt trauma patients presenting with no signs of life in the field. J Trauma Acute Care Surg. 2014;77:422-6.

[2] Flynn-O’Brien KT, Stewart BT, Fallat ME, et al. Mortality after emergency department thoracotomy for pediatric blunt trauma: analysis of the National Trauma Data Bank 2007-2012. J Pediatr Surg. 2016;51:163-7.

[3] Hill SJ, Zarroug AE, Ricketts RR, et al. Bedside placement of an aortic occlusion balloon to control a ruptured aorto-esophageal fistula in a small child. Ann Vasc Surg. 2010;24:822,e7-9.

[4] Norii T, Miyata S, Terasaka Y, et al. Resuscitative endovascular balloon occlusion of the aorta in trauma patients in youth. J Trauma Acute Care Surg. 2017; 82:915-20.

\section{Corresponding author:}

Alexis D Smith MD, Pediatric Surgeon, Children's Healthcare of Atlanta, Emory University, USA.

Email: alexisdeanasmith@gmail.com

(C) 2019 CC BY 4.0 - in cooperation with Depts. of Cardiothoracic/

Vascular Surgery, General Surgery and Anesthesia, Örebro

University Hospital and Örebro University, Sweden 